\title{
Mobility-Aided Routing in Multi-hop Heterogeneous Networks with Group Mobility
}

\author{
Leonardo Badia*, Nicola Bui ${ }^{\dagger}$, Marco Miozzo ${ }^{\dagger}$, Michele Rossi ${ }^{\dagger \S}$, Michele Zorzi ${ }^{\dagger \S}$, \\ *IMT Lucca, Institute for Advanced Studies, piazza S. Ponziano 6, 55100 Lucca, Italy \\ ${ }^{\dagger}$ Consorzio Ferrara Ricerche (CFR), via Saragat 1, 44100 Ferrara, Italy

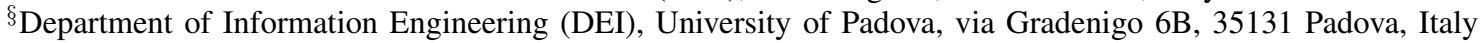 \\ Email: $\{$ bdalrd, buincl,mzzmrc\}@unife.it \{rossi,zorzi\}@dei.unipd.it
}

\begin{abstract}
This paper investigates routing strategies for mobile and heterogeneous multi-hop wireless networks. We leverage the knowledge about users mobility to improve the efficiency of route discovery and of the following data forwarding phase. In particular, we exploit group mobility behaviors, which allow us to apply a distributed on-line algorithm for the recognition of aggregated mobility patterns. Hence, we adopt a novel routing strategy that uses the aggregate structure formed within this algorithm to simplify the exchange of signaling and data messages. Finally, we demonstrate and quantify the benefits obtained with the proposed technique by means of a simulator for heterogeneous wireless networks.
\end{abstract}

\section{INTRODUCTION}

$\mathbf{M}$ ODERN telecommunications networks are moving towards extremely portable and wireless systems; in fact, the need for being connected anytime and everywhere is becoming more and more popular [1]. Modern laptops are usually equipped with multiple radio interfaces, e.g. IEEE802.11 and IEEE802.15.1 (Bluetooth) [2]. So a strong synergy of heterogeneous radio resources is expected in future generation networks. In this type of scenario environmental information as well as the coexistence of different radio technologies and network management entities will be exploited to improve performance.

In this paper, we study this research line, by discussing how to use the information about physical proximity and correlated mobility patterns within network protocols; in particular, we address the routing problem in scenarios characterized by strongly differentiated mobility [3]. Several research efforts have been devoted in recent years to different aspects of mobile networks, especially from the point of view of hierarchical routing [4], clustering of mobile nodes [5] and group mobility models [6]; our work integrates these topics.

Our main idea comes from an observation concerning human behavior. Usually people move in groups; hence, we can exploit this social habit to improve connectivity and QoS for those users with regular mobility patterns. Especially, the detection of aggregated structures can be useful in improving the performance of routing protocols. In fact, network topology changes due to mobility strongly affect routing policies. However, the awareness about mobility behaviors opens up the possibility of making predictions and improving management. Moreover, knowledge about local proximity of the nodes, recognizing aggregation into groups, can be exploited to fully benefit from the diversity of radio technologies.

This work has been supported by the WWI Ambient Networks Project. The views and conclusions contained herein are those of the authors and should not be interpreted as necessarily representing the Ambient Networks Project.
In this paper, we first discuss a method for representing these mobility behaviors, which accounts for correlated mobility patterns. Secondly, we propose a strategy to detect such relationships among mobile nodes. This results in a negotiation policy for the creation of aggregated structures called Routing Groups (RGs) [7], which consist of nodes having similar mobility patterns. This aggregation operation might be beneficial to take advantage of existing mobility structures and improve the efficiency in transmitting data and/or handling network related procedures, such as multiple simultaneous handovers of the RG members between access points (APs). In our approach, all messages going through the RG are managed by a single supervisor node, or gateway, called RG master. In this way, to successfully accomplish the handover procedure for a RG, instead of using a dedicated transmission for every terminal, a single message can be sent by the RG master for the whole RG. In general, this is true every time the information can be shared among users, that is, for all applications where a sort of multicast messaging is required or can be supported [8]. As a final original contribution, we propose to exploit the presence of group structures with a novel routing algorithm, obtained by modifying Dynamic Source Routing (DSR) [9]. This strategy, that we call DSR with $R G$-awareness (DSR-RG), imposes that the nodes in a RG route packets only through their RG master. In this way, local traffic is routed internally to the RG, whereas traffic towards further destinations is managed by RG masters only, which significantly decreases the congestion in the network. Notably, in the presence of multiple radio interfaces, it is also possible to exploit the RG paradigm by making use of the inherent parallelism among diverse radio interfaces, e.g., to route traffic within the $\mathrm{RG}$ and to/from outside the group. This technique has been evaluated and compared with the standard DSR protocol by means of extensive simulation runs.

The rest of the paper is organized as follows: in Section II we present the mobility model we adopt for the description of the group mobility behavior, in Section III we discuss the algorithm used to form routing groups and in Section IV we summarize the changes introduced to DSR. Section V describes our proprietary simulation tool and the scenarios we considered in the simulation campaign. In Section VI we present numerical results, while in Section VII we report the conclusions of our work.

\section{Mobility Model}

We consider the mobility model reported in [10], which is general and tunable to randomly generate a wide range of 
movement behaviors, whilst maintaining control over a set of mobility-related parameters.

The model obtains steady groups of mobile users. This is achieved by superimposing two different types of mobility: a random, independent component and another term which is representative of the cohesion among the users in the same group. This last term can be seen as an attraction force: an attractor node might be defined, which determines the direction of this attraction force.

We adopt a discrete-time model, updating the speed vector $\overrightarrow{v_{i}}$ differently for its magnitude and direction. For the absolute value of the speed at the $k$-th time step, $\tau_{k}$, a new sample $s_{k}$ is drawn from a Gaussian distribution and filtered with the current absolute value of the user's speed by a low-pass filter:

$$
\left|\overrightarrow{v_{i}}\left(\tau_{k}\right)\right|=(1-\zeta)\left|\overrightarrow{v_{i}}\left(\tau_{k-1}\right)\right|+\zeta s_{k},
$$

where $s_{k} \in N\left[\mu_{v_{i}}, \sigma_{v_{i}}\right]$, i.e., is Gaussian with mean value $\mu_{v_{i}}$ and standard deviation $\sigma_{v_{i}}$, and $\zeta \in[0,1]$ is the coefficient of the low-pass filter. Further, the direction of the speed $\vartheta_{i}$ is updated only after random time periods $\Delta \tau$, which are drawn with uniform distribution, i.e., $\Delta \tau \in U\left[\Delta \tau_{\min }, \Delta \tau_{\max }\right]$. Hence, upon obtaining $\Delta \tau, \vartheta_{i}$ is updated according to $\Delta \vartheta_{i}$, drawn with uniform distribution $U\left[-\vartheta_{\max } / 2, \vartheta_{\max } / 2\right]$ :

$$
\vartheta_{i}\left(\tau_{k}\right)=\vartheta_{i}\left(\tau_{k}-\Delta \tau\right)+\Delta \vartheta_{i}\left(\tau_{k}\right) \text {. }
$$

Finally, the speed is obtained as

$$
\overrightarrow{v_{i}}\left(\tau_{k}\right)=\left|\overrightarrow{v_{i}}\left(\tau_{k}\right)\right| e^{j \vartheta_{i}\left(\tau_{k}\right)} .
$$

The attraction component of the mobility is a function of the distance between the follower and its corresponding attractor. Also, we relate the attraction intensity to a specific parameter, called charge, which is representative of the strength of the group bond. Finally, we also include a variable $\beta$ that adds a random perturbation on how followers are attracted towards their attractor. Thus, the component of the speed due to the attraction force, $\overrightarrow{v_{a}}$, can be written as follows:

$$
\overrightarrow{v_{a}}\left(\tau_{k}\right)=\beta\left(\tau_{k}\right) C_{l} C_{f} d^{\alpha} \overrightarrow{u_{a}},
$$

where $C_{l}$ and $C_{f}$ are charges of the attractor and the follower, respectively, $d^{\alpha}$ reflects the dependence on the distance and $\overrightarrow{u_{a}}$ is the direction connecting the follower to the corresponding attractor. In (4) $\beta\left(\tau_{k}\right)$ is treated similarly to the absolute value of the independent component of the speed, i.e., every time unit a new sample is drawn from a Gaussian distribution and is filtered with the actual value. Hence, the resulting velocity can be calculated as the vector sum of the two speeds we found above:

$$
\vec{v}\left(\tau_{k}\right)=\overrightarrow{v_{i}}\left(\tau_{k}\right)+\overrightarrow{v_{a}}\left(\tau_{k}\right) .
$$

This model is quite general, as it is possible to use any existing random mobility model [6] to describe the independent component of the velocity. Moreover, it can be tuned to reflect different mobility behaviors, e.g., we can set the users to follow almost straight patterns or, vice versa, having rotatory behavior. Finally, we can control the way followers are attracted towards the corresponding attractors by fine tuning the parameters in (1)-(4); for example, it is possible to roughly define the average distance between users in a group by balancing the mean values of the distribution describing $\beta$ and $\left|v_{i}\right|$. Further details about the model and the mobility behaviors are available in [10].

\section{Aggregation Algorithm And Routing Strategy}

At first sight, the task of recognizing mobility patterns may seem difficult. In fact, a trivial approach is to observe how each node changes its position and assign a similarity value to each pair of nodes depending on how close these two nodes remain. However, this solution needs some information about the relative positioning of every pair of nodes. The message exchange necessary to gather and maintain this knowledge increases with the number of nodes and tends to saturate the radio capacity of the network.

We outline here an aggregation algorithm to gather and exploit information about mobility in order to form RGs (i.e., imposing a hierarchical structure usable by the routing algorithm) which does not need any knowledge about actual node positions. In order to properly perform aggregation, every user should be provided with a measure of the stability of the link between himself and his neighbors. To implement this, we let every user periodically broadcast a packet, named HELLO, containing information about his willingness to form a group with others. This message is also used to collect data about the HELLOs exchanged within the node's neighborhood. We transmit each HELLO after a random time interval with (tunable) average length equal to $T_{H E L L O}$. Randomness is required to reduce the message collision rate.

A stability measure is computed every $T_{S C A N}$ seconds (SCAN period) using the information gathered in the last $W$ SCAN periods. All transmitted HELLO messages will be correctly decoded by the receiver if the link from the transmitter is stable. Roughly speaking, this means that the receiver stays in coverage of the receiver and the environmental noise (e.g., fading, interference) does not degrade the useful signal too much. Since the number of messages that can be transmitted in $T_{S C A N}$ seconds is roughly $\left\lfloor T_{S C A N} / T_{H E L L O}\right\rfloor$, a link can be considered stable over the last $W$ periods if a user received $\eta W\left\lfloor T_{S C A N} / T_{H E L L O}\right\rfloor$ HELLO messages, where the parameter $\eta \in[0,1]$ allows a fine tuning of the stability condition, i.e., depending on the channel reliability. For example, with $\eta=0.8$ a link can be regarded as stable after the correct reception of $80 \%$ of the maximum number of HELLO packets that can be transmitted during the considered lapse of time.

Therefore, each user has to store in a variable the number of HELLOs received from any of its neighbors during the last $T_{S C A N}$ seconds. Let $R_{i j}^{k}$ represent the number of HELLOs received by user $i$ from user $j$ in the $k+1$-st SCAN period. Every user computes a measure of the stability of the link from any user he has correctly received a HELLO from (in the last $W$ periods) as follows:

$$
V_{i j}=\sum_{k=0}^{W-1}\left[R_{i j}^{k}\left(\eta W\left\lfloor T_{S C A N} / T_{H E L L O}\right\rfloor\right)^{-1}\right] .
$$

So $V_{i j} \geq 1$ means that link $j \rightarrow i$ has been stable over the last $W$ SCAN periods. Then each user counts how many links can be considered stable and stores this value into a further variable $S_{i i}$. Since this value carries the number of neighbors which have been within transmission range recently, it can be broadcast within the next HELLO packet as a measure of the suitability of making a group. Hence, every user $i$ collects the values $S_{i j}$ from any neighbor $j$ from which he receives a HELLO. $S_{i j}$ is the number of stable links of user $j$ as 
TABLE I

ROUTING GROUP FORMATION POLICY

\begin{tabular}{|c|c|c|c|}
\hline \multicolumn{3}{|c|}{ Condition } & Effect \\
\hline$S_{i i}<K$ & & & user $i$ does not form a group \\
\hline$S_{i i} \geq K$ & $\begin{array}{l}S_{i j} \geq K \\
S_{i j}<K \\
S_{i j} \geq K\end{array}$ & $\begin{array}{l}V_{i j} \geq 1 \\
V_{i j} \geq 1 \\
V_{i j}<1\end{array}$ & $\begin{array}{l}\text { user } i \text { forms a group } \\
\text { user } j \text { can be added to it } \\
\text { user } i \text { forms a group } \\
\text { user } j \text { can not be added to it } \\
\text { user } i \text { forms a group } \\
\text { link } j \rightarrow i \text { is not stable }\end{array}$ \\
\hline
\end{tabular}

measured by user $i$ (note that, due to communication delays, $S_{i j}$ might actually differ from the current value of $S_{j j}$ ). Now, it is possible to form the routing groups according to the rules illustrated in Table I, where $K$ is a threshold on the number of stable links needed to efficiently form a RG. As shown in the table, the introduction of user $j$ in the RG formed by user $i$ depends on three factors: $S_{i i}$, suitability to form a RG for $i, V_{i j}$, stability of link $j \rightarrow i$, and $S_{i j}$. In order to take advantage of the group structures within a routing protocol we elect a RG master for each formed group. To this end we use a procedure similar to the one in [11]. In fact, the basic idea is that all users belonging to the same group carry similar information for the routing protocol, so it is sufficient that one user per group participates in the procedure of route maintenance and setup. After the routing group procedure has started, each user broadcasts, within each HELLO message, a weight representing its suitability for the role of master. During the setup phase, every user considers itself as a master, then, once the routing group has been formed, each user elects as his master the one which is a master and has the highest weight in his group. In this way, any user that shares a stable link with another node, which better fits the role of master, elects this as his RG-master and becomes a RG member. If the link between a member and his RG-master fails, this member has to elect another master among its stable neighbors. If there are no stable neighbors or the user has the highest weight in his neighborhood, he elects himself as a RG-master.

\section{RG-AWARE DSR}

The Dynamic Source Routing (DSR) [9] algorithm is a reactive routing protocol. Whenever a node wants to send a message to a given destination, it starts a Route Discovery (RD) procedure. This operation consists of two phases. In the first phase the node sends a Route Request (RReq) message, which is broadcast to all nodes in the network. Eventually, this message reaches the intended destination which replies with a Route Reply (RRep), usually reversing the path traversed by the RReq.

In the presence of group mobility, this RD procedure suffers from the formation of sub-optimal paths; in fact, users moving together re-broadcast the same RD requests, thus they are likely to obtain similar paths for the same destination. In addition, when one of these paths fails, similar paths are usually affected as well. However, without a centralized awareness it is impossible to discover this in advance, but additional packets have to be sent, therefore causing a waste of resources in the network. Also, during the formation of these strongly correlated paths, a large number of redundant RReqs are replied, causing interference in the network and inducing a more severe contention at the MAC layer.
As a solution to these problems, we propose a modified version of DSR, called DSR with RG-awareness (DSR-RG), which exploits the existence of RG structures. As these structures are created and maintained based on the physical proximity of the nodes, we let all ordinary (i.e., non-master) members in a group not take part in the RD procedures. Instead, all nodes have to deliver their message to their RG master which in turn is the only node in the group in charge of the RD. With such a simple strategy, we can avoid part of the congestion in the network caused by RDs. In fact, for example, standard DSR has to start a different RD per each RG member, while our strategy only performs one RD per group. Moreover, we can form groups by broadcasting HELLO packets at a lower power level than, e.g., in the IEEE802.11 standard, so we can roughly control the size of the group by just limiting the transmission power of HELLO messages. In this way, not only do we reduce the interference introduced in the network, but we can also manage the communications within a group in a low power fashion. This also increases the capacity of the network; i.e., given the lower interference, two close groups can still generate intra-group traffic simultaneously.

Finally, as a further advantage, the RG awareness allows a reduction of the interference that inter-group communication may cause to long routes. This is achieved by assigning lower power to packets sent by RG members, which only need to communicate with their masters (short range transmissions). In addition, we can think of exploiting multiple radio interfaces. For example, if a short-range radio interface meant for PAN communication, such as Bluetooth or Zigbee, is available, we can think of exploiting it for inter-RG communication (between a member and its master). Instead, we leave a more powerful radio interface, e.g., a cellular or WLAN radio access technique, to cover the route from the RG master to the destination node. Of course, such a solution implies a higher hardware cost, as every terminal should own at least two radio technologies. However, the availability of multiple radio interfaces is common in current network terminals and is also well justified by the advantages in terms of increased network parallelism, decreased interference and overall better performance, as we show in Section VI.

\section{Simulation Framework}

This section gives a brief description of our event-driven network simulator for heterogeneous wireless system. A more detailed description can be found in [12]. The simulation tool used here has been specifically designed to model a multitechnology wireless communication scenario, where both mobile users and fixed access points (APs) coexist and communicate through the wireless medium. Node mobility, wireless channel variability and inter-user/inter-system interference have been explicitly accounted for. A simulated node may be either mobile or static, and behave as a user or an AP. In the following, we browse the node structure by providing a short description for each part.

The channel is modeled by accounting for path loss, shadowing and multi-path fading phenomena and using their product as the link gain, which is subsequently associated with each transmission link (a transmission link exists between each pair of nodes in the simulation). Path loss is implemented according to the well known Hata model [13]. Shadowing is accounted for through the Gudmundson model [14] and 
multi-path fading is implemented for each link through a Jakes simulator with programmable number of oscillators [15].

The physical layer entity takes as input the channel gain matrix created and maintained by the channel module and the transmission powers selected by each user and returns Signal to Interference plus Noise Ratio (SINR) metrics for each active link. The current version of the simulator implements a physical layer module for the IEEE802.11b and the IEEE802.15.4 (ZigBee) radio levels [2]. Errors on the transmitted data streams can be tracked at the bit level and coding can also be accounted for using pre-computed coding gain curves [16]. Both IEEE802.11b and IEEE802.15.4 radio levels implement a receiving model which accounts for possible interfering transmissions during a packet reception. Therefore, simultaneous transmissions do not lead to a certain collision at the receiver. In this way, we can effectively account for the capture effect [17] at the physical layer.

The MAC level of IEEE802.11b implements the Distributed Coordination Function (DCF) technique as specified by the standard [2]. For what concerns the Collision Avoidance feature, both Basic Access Mode and RTS-CTS mode are possible. For the IEEE802.15.4 technology we implemented the beaconless peer-to-peer mode. This mode handles transmissions similarly to IEEE802.11, i.e., every node can transmit after winning the contention for the shared channel.

The routing level covers an important role in the simulator. Currently, this level includes a variant of the Dynamic Source Routing (DSR) algorithm [9] with the capability of routing packets across different technologies and exploiting the RG structure as well as the standard DSR implementation.

For the mobility we implemented both independent and group mobility behaviors, according to the model in Section II. Every simulated entity can be static or mobile and, in this last case, can either move independently or in a group fashion.

For what concerns the simulation scenario, we evaluate the impact of the RG structures testing our proposed algorithm on a mobile wireless heterogeneous network. In particular, we consider a network with one static access point, randomly placed within the simulation area, and 30 mobile users, 10 of which move independently, while the remaining 20 are equally distributed among 4 mobility groups. All users are equipped with IEEE802.11b and IEEE802.15.4 (ZigBee). The former radio interface is used to deal with the data traffic and the routing protocol, while the latter is used to manage the RG formation algorithm, that is the transmission of HELLO packets. Note that the two radios operate on non-overlapping bands as we selected the $868 \mathrm{MHz}$ band for ZigBee, while IEEE 802.11b operates in the $2450 \mathrm{MHz}$ band. In particular, data flows are sent using different power levels for intra- and extra-group communications. In fact, since it is possible to correctly deliver a message within the group using only a fraction of the maximum power, we can use full power only for the packets routed outside the group, thereby limiting the interference due to intra-group traffic.

The scenario focuses on the comparison between DSR-RG, presented above, and standard DSR. For instance, we monitor performance metrics like throughput, delays, energy consumption when the traffic within the network is generated only by RG members (we have an increasing number of members transmitting simultaneously per group with a growing packet generation rate).
We consider a pedestrian mobility scenario; so we let the users speed range from 0.5 to $1.2 \mathrm{~m} / \mathrm{s}$ and we set the attraction force so as to maintain the average distance between any two users in the same group less than the transmission range of ZigBee and IEEE802.11 at minimum power level (approximately equal to $15 \mathrm{~m}$ ).

\section{RESUlTS}

We compare DSR and DSR-RG for the cases of 1,2 and 4 data flows taking place in each group. Each point of the graphs is the average of at least 30 simulation runs, consisting of 600 seconds of simulated time, which guarantees that $95 \%$ confidence intervals are within $12 \%$ of the average values we show in the graphs.

Fig. 1a shows the average throughput per active RG member as a function of the packet generation rate. From this figure we can see that our method outperforms standard DSR; this is mainly due to the lower congestion introduced by $\mathrm{RD}$ procedures. In fact, all RG members do not add any traffic in the network for the routing protocol (RReqs and RReps). Moreover, when there is more than one active flow per mobility group, we observe a further improvement. The reason for this is that the nodes belonging to the same group entrust the same RG master to deliver their packets, so only the master has to perform RDs. Although DSR-RG has better performance than standard DSR for low to medium packet generation rates, at higher rates the performance degrades, becoming similar to that of the original DSR. This is mainly due to the bottleneck which is inherent in sending the whole RG traffic to a single node (RG master). In fact, RG structures are useful until the load of the master overcomes its capacity. Fig. 1b shows the impact of our techniques on the energy consumption: DSR-RG outperforms DSR in every situation albeit having to broadcast HELLO packets. This gain is mainly due both to the lower power level used for intra-group transmissions and to the limited impact of HELLO messaging on the energy metric (as compared to the consumption of RDs).

In Fig. 1c we plot the average delay to successfully deliver a packet. The benefit realized for this metric is evident and can be better explained examining Fig. 1d, where the average number of RDs to correctly deliver a packet is reported as a function of the packet generation rate. Thanks to our algorithm we can exploit the correlation (in terms of physical proximity) among users in the same group, so we need a lower number of RDs.

Finally, Fig. 1e shows the average number of transmissions needed to reach the destination. DSR algorithm is more likely to use shorter paths, whereas our solution produces slightly longer routes, basically due to the first hop to the RG leader. However, we also observe that, as the number of flows increases, the average length of routes produced by DSR further decreases. This is due to the need to manage the increasing congestion due to the growing number of control packets related to the RD procedures and the users data traffic. The effect on our scheme is instead only marginal as a single $\mathrm{RD}$ per group is needed.

To sum up, it is possible to say that the additional price to pay for an additional radio interface for every node, which is however generally supported by modern wireless terminals and current network deployments, can be well justified by the 


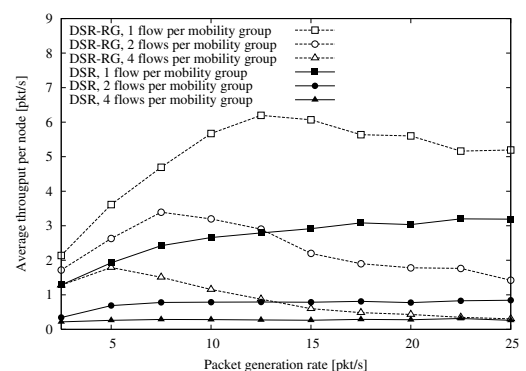

(a) Average throughput per active node.

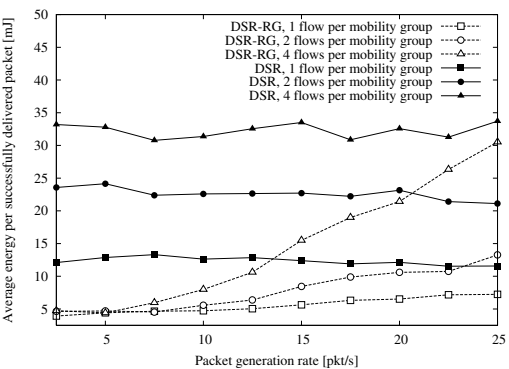

(b) Average energy spent per packet successfully delivered.

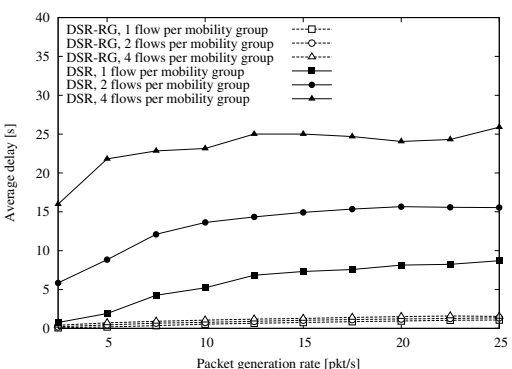

(c) Average delay.

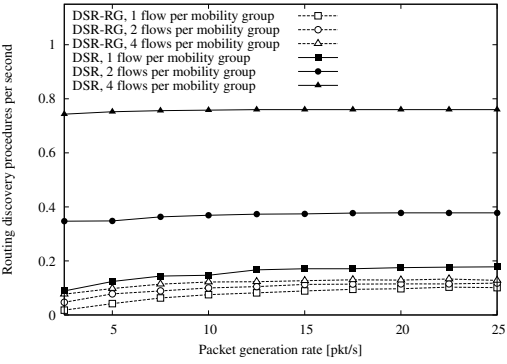

(d) Average number of Route Discovery procedures.

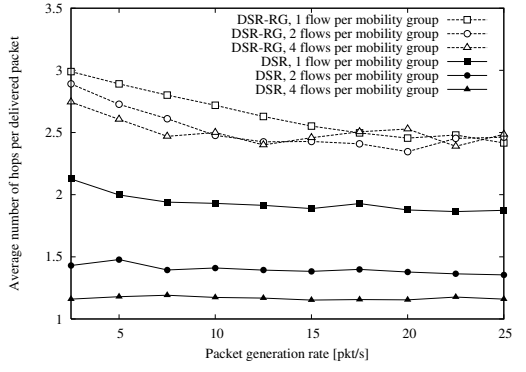

(e) Average number of hops needed to reach the destination

Fig. 1. Comparison between DSR and DSR-RG for 1,2,4 flows per mobility group as a function of the packet generation rate $\lambda$.

obtained advantages in terms of decreased delay and energy expenditure, as well as overall higher throughput. Moreover, note that the additional overhead required by our algorithm to collect and exploit RG information is not very high, as all the results are obtained broadcasting HELLO packets every $T_{H E L L O}=10$ seconds. Finally, the choice of DSR as the basic algorithm on top of which we apply RG considerations is by no means fundamental. We can in fact see the $R G$ awareness as an additional feature, which can be combined with any other algorithm to improve network performance.

\section{CONCLuSiOnS}

In this paper we discussed the impact of mobility factors on routing strategies, focusing in particular on the aggregated mobility behavior of wireless terminals, which may lead to the identification of grouped structures named Routing Groups, whose existence can be exploited for example at the routing protocol level.

To this end, we first described a general and tunable group mobility model. Furthermore, we developed a distributed online aggregation algorithm and a routing group aware variant of the DSR protocol. Then, we described a simulator capable of representing the behavior of mobile, wireless and multitechnology networks that we used to compare standard DSR and our RG-aware variant. We performed an extensive simulation campaign to highlight, even in simple scenarios, the benefits obtained by exploiting the presence of group mobility.

Future research can be devoted to the extension of these results to other routing strategies, including for example hierarchical and planar routing, or implementing advanced techniques to manage Routing Groups, such as load balancing within the group or dynamic election of the masters according to their status in terms of position, coverage and battery level.

\section{REFERENCES}

[1] The Ambient Networks project, http://www.ambient-networks.org/.

[2] IEEE 802 LAN/MAN Standards Committee documentation, http://www.ieee802.org.

[3] M. Zonoozi and P. Dassanayake, "User mobility modeling and characterization of mobility patterns," IEEE Journal on Selected Areas in Communications, vol. 15, pp. 1239-1252, 1997.

[4] K. Xu and M. Gerla, "A heterogenous routing protocol based on a new stable clustering scheme," in Proceedings IEEE MILCOM, vol. 2, Oct 2002, pp. 838-843.

[5] S. Basagni, M. Mastrogiovanni, A. Panconesi, and C. Petrioli, "Localized protocols for ad hoc clustering and backbone formation: A performance comparison," in IEEE Transactions on Parallel and Distributed Systems, vol. 17, no. 4, Apr 2006, pp. 292-306.

[6] T. Camp, J. Boleng, and V. Davies, "A survey of mobility models for ad hoc network research," Wireless Communications and Mobile Computing, vol. 2, pp. 483-502, 2002.

[7] Y.-L. Chang and C.-C. Hsu, "Routing in wireless/mobile ad-hoc networks via dynamic group construction," Springer Journal Mobile Networks and Applications, vol. 5, no. 1, pp. 27-37, Mar. 2000.

[8] C. K. Toh, "Associativity-based routing for ad-hoc mobile networks," Kluwer Wireless Personal Communications, vol. 4, pp. 103-139, 1997.

[9] D. B. Johnson, D. A. Maltz, and Y.-C. Hu, "The dynamic source routing protocol for mobile ad hoc networks (DSR)," 2003, available as Internet draft, draft-ietf-manet-dsr-09.txt.

[10] L. Badia and N. Bui, "A group mobility model based on nodes' attraction for next generation wireless networks," in Proceedings IEE Mobility, Bangkok, Thailand, Oct 2006.

[11] S. Basagni, "Distributed and mobility-adaptive clustering for multimedia support in multi-hop wireless networks," in Proceedings of IEEE VTC Fall, vol. 2, 1999, pp. 889-893.

[12] L. Badia, N. Bui, M. Miozzo, M. Rossi, and M. Zorzi, "On the exploitation of user aggregation strategies in heterogeneous wireless networks," in Proceedings CAMAD, Trento, Italy, June 2006, pp. 8-15.

[13] A. Goldsmith, Wireless Communications. Cambridge Univ. Press, 2005.

[14] G. L. Stüber, Principles of Mobile Communication. Kluwer Academic Publishers, 2003.

[15] W. C. Jakes, Microwave Mobile Communications. Wiley-IEEE, 1994.

[16] V. Atanasovski and L. Gavrilovska, "Influence of header compression on link layer adaptation in IEEE 802.11b," in Proceedings IWCMC, vol. 2, 2005, pp. 1551-1556.

[17] J. H. Kim and J. J. Lee, "Capture effects of wireless CSMA/CA protocols in Rayleigh and shadow fading channels," in IEEE Transactions on Vehicular Technology, vol. 48, no. 7, Jul 1999, pp. 1277-1286. 